\title{
ESPECIE NUEVA DE COELIOXYS \\ (HYMENOPTERA: APOIDEA: MEGACHILIDAE) PARA LA HISPANIOLA
}

\author{
Julio A. Genaro
}

Investigador Asociado, York University, Department of Biology, 4700 Keele Street, Toronto, Ontario, M3J 1P3, Canada.polimita@hotmail.com

\section{RESUMEN}

Se describe una especie nueva de Coelioxys, subgénero Cyrtocoelioxys, para La Hispaniola. Esta constituye la segunda especie del género conocida para la isla. Esta especie está muy relacionada con $C$. alayoi Genaro, de Cuba. Se presenta una lista anotada de las especies antillanas de Coelioxys (Cyrtocoelioxys).

Palabras clave: Hymenoptera, Apoidea, Megachilidae, Coelioxys, Cyrtocoelioxys, especie nueva, La Hispaniola.

\section{ABSTRACT}

A new species from Hispaniola of the genus Coelioxys, subgenus Cyrtocoelioxys is described. This is the second known species of the genus from the island. It is closely related to C. alayoi Genaro from Cuba. An annotated checklist of the Antillean species of the subgenus Cyrtocoelioxys is presented.

Key words: Hymenoptera, Apoidea, Megachilidae, Coelioxys, Cyrtocoelioxys, new species, Hispaniola.

\section{INTRODUCCIÓN}

El género Coelioxys está compuesto por abejas con pelos en los ojos, tegumento negro y en ocasiones con algunas áreas ferruginosas. El cuerpo está provisto de poca pubescencia, debido a sus hábitos parasíticos, ya que cleptoparasitan fundamentalmente abejas del género Megachile (Baker, 1975; Mitchell, 1973; Michener et al., 1994; Michener, 2000).

El género se ha estudiado bastante: Friese (1921) publica sobre las formas Neotropicales; Mitchell (1962) redescribe, presenta una clave e ilustra las especies del este de los Estados Unidos; Mitchell (1973) revisa los subgéneros del Hemisferio Occidental; Baker (1975) estudia las especies pertenecientes a cinco subgéneros Neárticos. Toro y Fritz (1993) publican sobre las abejas argentinas del subgénero Cyrtocoelioxys; Genaro (1998) esclarece la localidad tipo de C. (Cyrtocoelioxys) tridentata (Fabr.) y sinonimiza a C. uhleri Cresson con ésta; Genaro (2001) describe dos especies nuevas para Cuba, de los subgéneros Boreocoelioxys y Cyrtocoelioxys; Nagase (2006) estudia las especies de Japón. Moure et al. (2007) publican el catálogo de las abejas Neotropicales, ofreciendo datos de distribución y nomenclatura; Pérez-Gelabert (2008) compila el conocimiento sobre los artrópodos de La Hispaniola, brindando información sobre las abejas.

Las abejas incluidas en Coelioxys son muy uniformes en su apariencia, como ejemplo basta decir que desde la descripción del género por Latreille (1809) ninguna abeja descrita (ahora considerada en Coelioxys) se ha incluido en otro género (Baker, 1975). Sin embargo, las especies se diferencian bien por la variación en la forma del escutelo, el extremo del abdomen y la esculturación.

Para La Hispaniola solo se conoce a C. (Neocoelioxys) vigilans F. Smith, 1879, una especie comunmente observada (Genaro, 2007). A continuación se describe una segunda especie, la cual pertenece al subgénero Cyrtocoelioxys.

Se revisaron las colecciones entomológicas de las siguientes instituciones: CUBA, Museo Nacional de Historia Natural, Ciudad de La Habana; Instituto de Ecología y Sistemática, Ciudad de La Habana. REPÚBLICA DOMINICANA, Museo Nacional de Historia Natural, Santo Domingo; colección de Eugenio De Jesús Marcano, Universidad Autónoma de Santo Domingo. PUERTO RICO, Museo de Entomología y Biodiversidad Tropical, Estación Experimental Agrícola en Río Piedras; Departamento de Biología, Universidad de Puerto Rico, Mayagüez. ESTADOS UNIDOS, American Museum of Natural History, Nueva York; Academy of Natural Sciences of Philadelphia, Philadelphia; Florida State Collection of Arthropods, Gainesville, Florida (FSCA); Museum of Comparative Zoology at Harvard University; 
Natural History Museum, University of Kansas; United States National Museum, Smithsonian Institution (USNM). CANADA, Canadian National Collection of Insects, Arachnids and Nematodes, Ottawa.

La terminología utilizada para definir las estructuras sigue en parte a Michener et al. (1994) y Mitchell (1973), y para la escultura de la superficie del tegumento se sigue a Harris (1979). Las abreviaturas son usadas como sigue: cada tergo o esterno metasomal es llamado $\mathrm{T}$ o $\mathrm{S}$, respectivamente, seguido por un número que lo define específicamente, ejemplo: T1 es el primer tergo metasomal y S1 el primer esterno. La densidad de las puntuaciones está dada en términos de la relación entre el diámetro de la puntuación (d) y el espacio (e) entre ellas, como ejemplo e $=2 \mathrm{~d}$, para demostrar su separación. Las observaciones, medidas y descripciones se realizaron con un microscopio estéreo marca Wild M3C. Las mediciones fueron hechas con un micrómetro ocular, mientras que las fotografías fueron preparadas con el sistema de procesamiento de imágenes Microptics ML-1000.

\section{RESULTADOS \\ SISTEMATICA \\ Familia Megachilidae \\ Coelioxys (Cyrtocoelioxys) danielperezi sp. nov.}

(Figs. 1-9)

Diagnosis. Ambos sexos negros, excepto tarsos, área central de la mandíbula, S1 y S6 castaño rojizo; margen posterior del escutelo angulado medialmente (redondeado en C. alayoi). Hembra: T6 con un diente medio apical dirigido hacia arriba (Fig. 4), S6 en forma de U, marginado con pelos, y un diente central corto (Fig. 5). Macho: T6 con procesos dorsales aplanados, cortos, ligeramente divergentes, ápices redondeados; excavación dorsal estrecha, alargada; procesos ventrales divergentes hacia ápice, espinosos; procesos laterales espinosos (Fig. 9).

Diagnosis. Both sexes black, except tarsi, central area of mandibles, S1 and S6 reddish brown; posterior margin of scutellum medially angulated (rounded in C. alayoi). Female: apex of T6 with spine curved upward (Fig. 4), S6 U shaped, fringed with hairs, and a short, central spine (Fig. 5). Male: T6 with dorsal processes short, flattened, slightly divergents, apices rounded; dorsal excavation narrow, elongate; ventral processes diverging to apex, spinoses; lateral processes spinoses (Fig. 9).
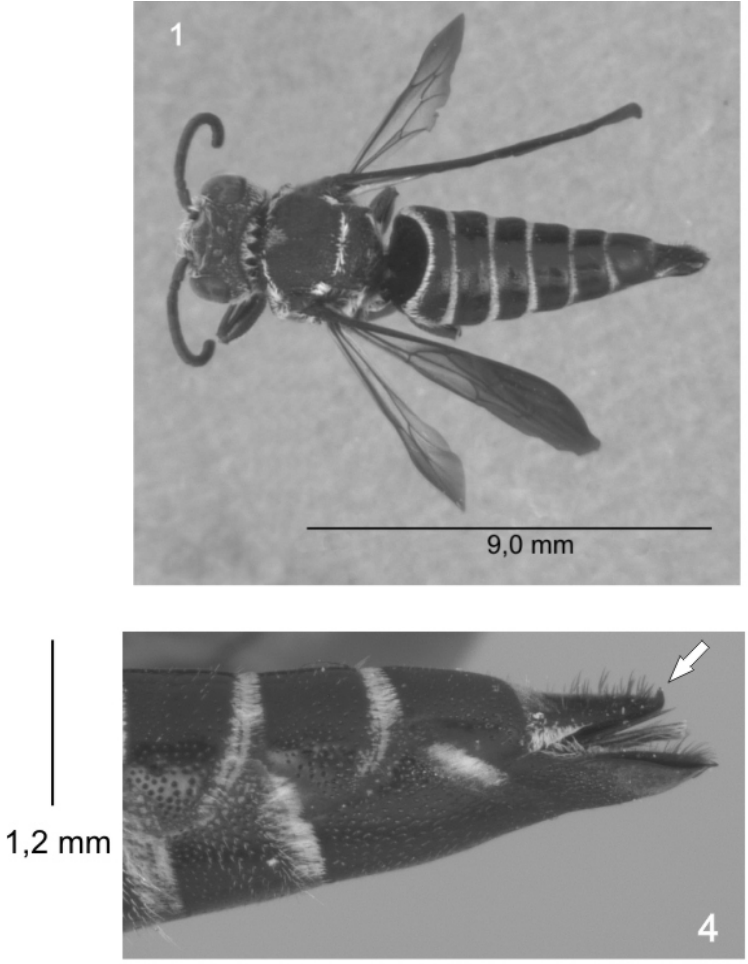
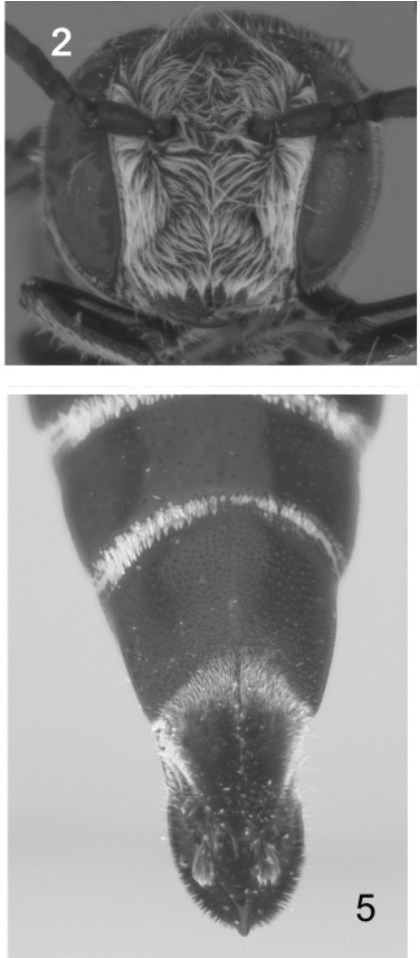

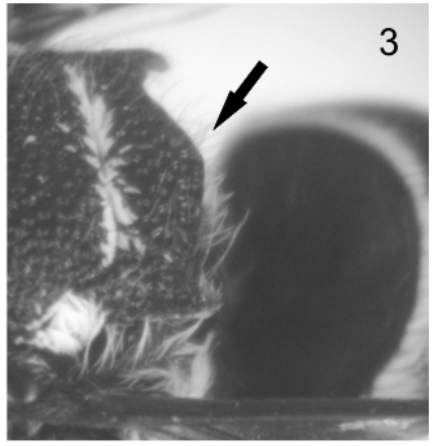

Figuras 1-5.

Coelioxys (Cyrtocoelioxys) danielperezi sp. nov., hembra. 1-Vista dorsal. 2-Cabeza en vista frontal. 3-Escutelo y axilas. 4Vista lateral del final del metasoma (la flecha muestra diente sobre T6). 5-Vista dorsal del final del metasoma mostrando T6. 
Descripción. Hembra. Longitud corporal total: 9,8-11,3 mm. Longitud ala superior: 5,9-7,0 mm.

Coloración. Tegumento negro (Fig. 1), excepto ápice y borde mandíbulas, tarsos de todas las patas, S1 y S6 castaño rojizo. Alas con venación negra; ala superior más oscurecida en celda marginal, celdas submarginales, segunda celda medial y ápice; ala inferior más oscurecida en ápice.

Pubescencia. Blanca, apretada en las siguientes áreas: cabeza, clípeo, área supra-clipeal, supra-antenal (Fig. 2) y genal, más espesa en área paraocular. Mesosoma: margen anterior, posterior y línea media escudo, margen anterior escutelo, área cresta escutelar, márgenes mesepisterno, coxa, área externa de fémur y tibia, y fascia metasomal. Pelos erectos, blancos, aislados en vértex, escudo y escutelo. Pelos amarillos en tarsos. Área apical de T6 cubierta de pelos amarillos erectos. Margen apical de S6 con setas amarillas, gruesas, muy unidas, cortas.

Estructura. Doce segmentos antenales. Mandíbula tridentada; margen externo curvado, diente medio ligeramente más cercano al diente apical; diente interno más corto que el medio. Área supra-antenal algo plana. Surco medio dorsal del escutelo, variable. Área media del margen posterior del escutelo ligeramente angulado (Fig. 3). T6 estrecho posteriormente; área apical en forma de V, con depresiones laterales subapicales, separadas por surco medio; extremo apical con diente dirigido hacia arriba (Fig. 4). S6 en forma de U, marginado con pelos y un diente central corto (Fig. 5).

Esculturación. Cabeza: puntuaciones gruesas en gena y vértex, más esparcidas alrededor de ocelos; puntuaciones alargadas en área supra-antenal (debajo ocelo medio); puntuaciones menores, más unidas (e $<$ d) en clípeo, área paraocular y supraclipeal. Mesosoma: escudo, escutelo y axila puntuado-reticulado, puntuaciones de igual tamaño a los del vértex; tégula imbricada, con algunas puntuaciones aisladas. Metepisterno con algunas puntuaciones muy aisladas. Metasoma: T1 densamente puntuado, con puntuaciones irregulares $(\mathrm{e}=0,5-2 \mathrm{~d})$. Área basal de $\mathrm{T} 6$ densamente puntuada, con puntuaciones más pequeñas irregulares $(\mathrm{e}<\mathrm{d} ; \mathrm{e}=0,5-1 \mathrm{~d})$. Área pregradular de T4 y T5 con puntuaciones intermedias, algo mayores que las de T6 y algo menores que las de T2-T3. Área pregradular de T2-T5 irregular y densamente puntuada $(\mathrm{e}=0,5-2 \mathrm{~d})$; área pregradular con puntuaciones aisladas. $\mathrm{S} 1$ con puntuaciones irregulares $(\mathrm{d}>\mathrm{e}$; $\mathrm{e}=0,5-2 \mathrm{~d}$ ), puntuaciones grandes aisladas entre otras menores. Superficie media de S1 elevada, con surco. $\mathrm{S} 2-\mathrm{S} 5$ con puntuaciones irregulares $(\mathrm{e}=0,5-2,5 \mathrm{~d})$, más pequeñas en $\mathrm{S} 5 ; \mathrm{S} 6$ impuntuado en área apical.

Macho. Similar a la hembra excepto en lo siguiente: longitud corporal total: $9,8 \mathrm{~mm}$. Longitud ala superior: $6,3 \mathrm{~mm}$
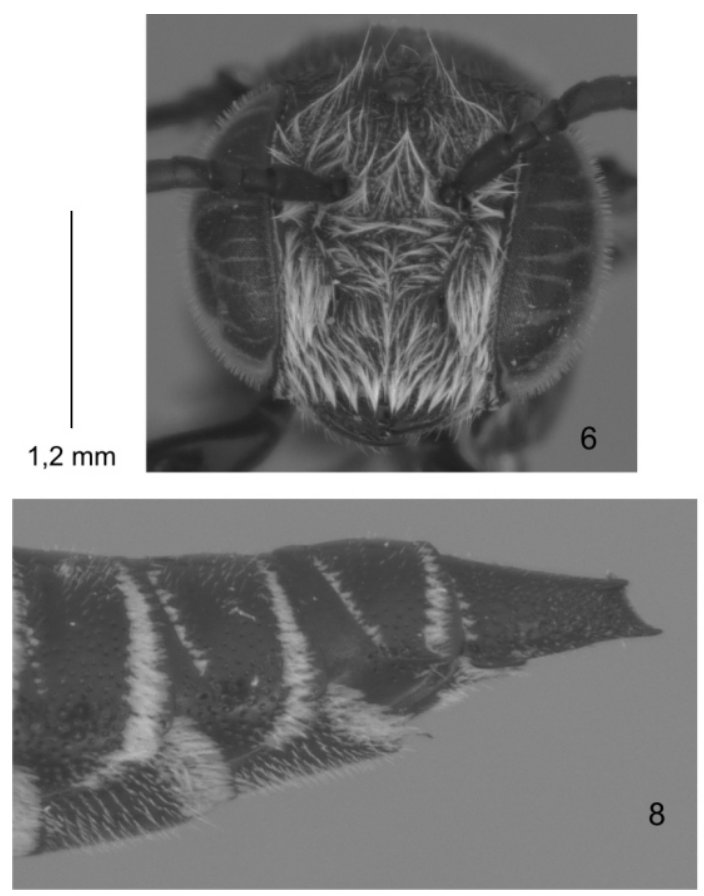
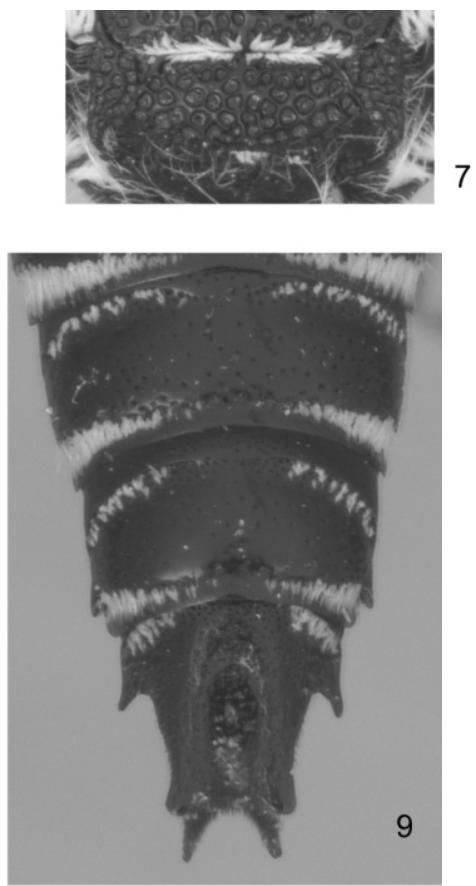
Pubescencia. Concavidad hipostomal desnuda. T6 con pubescencia amarilla.

Estructura. Antena con 13 segmentos. La Figura 8 muestra a T6 en vista dorsal, notándose los procesos dorsales y ventrales. T6 con procesos dorsales aplanados, cortos, ligeramente divergentes, ápices redondeados; excavación dorsal estrecha, alargada; procesos ventrales divergentes hacia ápice, espinosos; procesos laterales espinosos (Fig. 9). Abultamiento central de S1 menor.

Esculturación. Surco medio dorsal del escutelo, desarrollado. T1 irregularmente puntuado (e=0,5-3d). T4-T6 con puntuaciones algo más pequeñas; T2-T5 con zona del disco impuntuada y algunas puntuaciones aisladas, a los lados con puntuaciones irregulares $(d>e ; e=0,5-2 d)$. T6 irregularmente puntuado $(d>e$; $\mathrm{e}=0,5-4 \mathrm{~d}$ ); puntuaciones grandes aisladas entre puntuaciones más pequeñas y densas. Área postgradular de S5 poco esclerotizada, con puntuaciones muy unidas $(\mathrm{d}>\mathrm{e})$, disminuyendo hacia ápice.

Tipos. Holotipo hembra y macho alotipo. HISPANIOLA: Dominican Republic, RD-244 Cerro Santo Tomás, Parque Botánico de Jánico, Santiago province, 493 m, 19¹9382' N7049.240'W, 27.IV.2004, cols. D. Pérez, B. Hierro y R. Bastardo (USNM). Paratipos hembras. HISPANIOLA: Dominican Republic RD277, Guaraguao, Parque Nacional de Este, La Altagracia province, $18^{\circ} 20.296^{\prime} \mathrm{N} 68^{\circ} 48.907^{\prime} \mathrm{W}$, near sea level, 19-20.VII.2004, col. D. Pérez (USNM); RD-192, road down from door, 1000-800 m, Parque Nacional Sierra de Bahoruco, Independencia province, 12.xii.2003, cols, D. Pérez, R. Bastardo y B. Hierro (FSCA).

Etimología. Dedicada a Daniel E. Pérez-Gelabert por su valiosa contribución a la ampliación del conocimiento de los invertebrados antillanos y en especial a los de La Hispaniola.

Comentario. La única especie antillana del subgénero Cyrtocoelioxys donde la hembra presenta T6 con un diente medio apical dirigido hacia arriba es C. alayoi, de Cuba. Sin embargo, esta especie aparentemente es más robusta. Aunque debe tenerse en cuenta que se conoce sólo de una hembra, y el tamaño es una expresión del tamaño del hospedero. C. alayoi tiene más áreas del tegumento castaño rojizo como: tégula, patas, esternos y área lateral de T1. Los machos de C. alayoi se desconocen. La otra especie de este género conocida anteriormente para La Hispaniola pertenece a otro subgénero, Neocoelioxys, y cumple con las caraterísticas que lo definen, como por ejemplo macho con T6 con cuatro espinas agudas y hembra con una muesca a cada lado, cerca del ápice, terminando en un proceso lanceolado.

Era de esperar que existieran otras formas de Coelioxys en La Hispaniola, ya que su hospedero, Megachile, presenta unas 11 especies (Genaro, 2007), y Cuba, una isla con características ecológicas similares, tiene seis especies de Coelioxys. Pueden existir otras especies, pero al igual que en Cuba estas deben aparecer en niveles poblacionales muy bajos. Solamente C. rufipes Guérin-Méneville, 1845 es abundante en Cuba, como sucede con C. vigilans en La Hispaniola (decenas de ejemplares examinados en colecciones siempre pertenecieron a esta última especie).

Hace unos 33 a 35 millones de años los núcleos de las futuras Antillas estuvieron unidos entre sí, lo que permitió el intercambio de la fauna. Posteriormente, a partir de los 14 a 16 millones de años estas tierras comenzaron a separarse, quedando las poblaciones aisladas (Iturralde-Vinent y MacPhee, 1999; IturraldeVinent, 2006). El escenario observado desde finales del Eoceno hasta el Oligoceno Superior demuestra la interacción de la biota en las Proto Antillas y que la historia geológica de Cuba y La Hispaniola estuvo muy relacionada, llegando a estar unidas durante mucho tiempo, en momentos de condiciones favorables para sostener la biota. Debido a fenómenos de surgimiento e inmersión, las tierras quedaron separadas, pero cada isla mantuvo los ancestros, de los cuales muchos evolucionaron hacia formas diferentes. En este caso, he encontrado especies de abejas y avispas muy afines, con contrapartes vicariantes como por ejemplo: Coelioxys alayoi vs $C$. danielperezi sp. nov; Triepeolus cuabitensis Genaro, 2001 vs Triepeolus sp.; Hoplisoides jaumei (Alayo, 1969) vs Hoplisoides sp.; Isodontia poeyi Pate, 1948 vs Isodontia sp. La fauna de invertebrados de La Hispaniola está poco documentada, por lo que estas especies necesitan aún de un estudio más profundo y de su descripción. El estudio de Pérez-Gelabert (2008) constituye la base de lo hecho y debe estimular su desarrollo.

\section{LISTA DE LAS ESPECIES DE COELIOXYS (CYRTOCOELIOXYS) DE LAS ANTILLAS}

alayoi Genaro, 2001 (=sp. A. Alayo, 1976). Cuba.

danielperezi sp. nov. Hispaniola.

sp. Puerto Rico. Para comentarios sobre esta especie vea Genaro y Franz (2008).

spinosa Dewitz, 1881. Puerto Rico.

tridentata (Fabricius, 1775) (= C. uhlerii Cresson, 1865). Cuba. 


\section{AGRADECIMIENTOS}

A Daniel Pérez-Gelabert por permitir estudiar las colectas de abejas realizadas durante su proyecto en la República Dominicana. A David Furth y Brian Harris (USNM) por facilitar el acceso al material estudiado. Antonia Guidotti (Royal Ontario Museum, Toronto) amablemente permitió el uso del sistema para obtener imágenes. La organización Idea Wild proporcionó equipamiento que permitió y facilitó el examen microscópico de los ejemplares.

\section{LITERATURA CITADA}

Baker, J. R. 1975. Taxonomy of five Neartic subgenera of Coelioxys (Hymenoptera: Megachilidae). Univ. Kansas Sci. Bull., 50: 649-730.

Friese, H. 1921. Über die kegelbienen (Coelioxys) Brasiliens. Zool. Jahrbüchern, 44: 421-486.

Genaro, J. A. 1998. Distribution and synonymy of some Caribbean bees of the genera Megachile and Coelioxys (Hymenoptera: Megachilidae). Caribbean J. Sci., 34: 151-152.

Genaro, J. A. 2001. Especies nuevas de abejas de Cuba y la Española (Hymenoptera: Colletidae, Megachilidae, Apidae). Revista de Biología Tropical, 49: 951-959.

Genaro, J. A. 2007. Las abejas (Hymenoptera: Apoidea: Anthophila) de la Hispaniola, Antillas. Boletín de la Sociedad Entomológica Aragonesa, 40: 247-254.

Genaro, J. A. y N. M. Franz. 2008. The bees of Greater Puerto Rico (Hymenoptera: Apoidea: Anthophila). Insecta Mundi, 40:1-24.

Harris, R. A. 1979. A glossary of surface sculpturing. Occasional Papers in Entomology, Department of Food and Agriculture, Division of Plant Industry California, 28: 131.

Iturralde-Vinent, M. A. 2006. Meso-Cenozoic Caribbean paleogeography: implications for the historical biogeography of the region. International Geology Review, 48: 791-827.

Iturralde-Vinent, M. A. y R. D. E. MacPhee 1999. Paleogeography of the Caribbean Region: implications for Cenozoic biogeography. Bulletin of the American Museum of Natural History, 238: 1-95.

Mitchell, T. B. 1962. Bees of the Eastern United States. II. North Carolina Agric. Exp. Sta. Tech. Bull., 152: $1-557$.

Mitchell, T. B. 1973. A subgeneric revision of the bees of the genus Coelioxys of the Western Hemisphere. Contr. Dep. Entomol. North Carolina State Univ. 124 pp.

Michener, C. D. 2000. The bees of the world. Johns Hopkins Univ. Press, Baltimore. 913 pp.

Michener, C. D.; R. J. McGinley y B. N. Danforth. 1994. The bee genera of North and Central America (Hymenoptera: Apoidea). Smithsonian Inst. Press, Washington, D. C. 209 pp.

Moure, J. S.; G. A. R. Melo y A. DalMolin. 2007. Megachilini Latreille, 1802. Pp: 917-1001. En: Moure, J. S.; D. Urban y G. A. R. Melo (editors). Catalogue of bees (Hymenoptera, Apoidea) in the Neotropical region. Sociedade Brasileira de Entomologia, Curitiba. 1058 pp.

Nagase, H. 2006. Synopsis of the bee genus Coelioxys Latreille (Hymenoptera: Megachilidae) of Japan, with description of a new species. Entomological Science, 9: 223-238.

Pérez-Gelabert, D. E. 2008. Arthropods of Hispaniola (Dominican Republic and Haiti): a checklist and bibliography. Zootaxa, 1831:1-530.

Toro, H. y M. Fritz. 1993. Las especies argentinas del género Coelioxys (Cyrtocoelioxys) (Hymenoptera: Megachilidae). Acta Entomológica Chilena, 18: 147-161. 